\title{
SISTEMAS DE INFORMACIÓN GEOGRÁFICA: APLICACIÓN PRÁCTICA PARA EL ESTUDIO DE ATROPELLOS EN EL CERCADO DE LIMA, PERÚ
}

\author{
Akram Hernández-Vásquez ${ }^{1, a, b}$, Diego Azañedo 2,c, Guido Bendezú-Quispe ${ }^{3, d}$, Josmel Pacheco-Mendoza ${ }^{4, e, f,}$ \\ R. Martín Chaparro ${ }^{1, g, h}$
}

\section{RESUMEN}

El objetivo del estudio fue explorar geoespacialmente los patrones de ocurrencia de atropellos en el Cercado de Lima, Perú. Se describieron y georreferenciaron los atropellos registrados en el Censo Nacional de Comisarías 2015 del Instituto Nacional de Estadística e Informática. Posteriormente, se realizó un análisis Kernel Density para localizar áreas con alta, mediana y baja densidad de eventos. Se estudiaron 171 registros de atropellos, los tipos de vehículo involucrados fueron automóvil $(56,7 \%)$ y vehículos menores $(22,8 \%)$. El mayor porcentaje de atropellos $(38,6 \%)$ ocurrió entre las 12.00-17.00 horas. Se encontraron dos zonas de alta densidad y dos de densidad intermedia para atropellos, coincidiendo con ubicaciones reportadas previamente como críticas por sus deficiencias y mayor probabilidad de accidentes de tránsito. El empleo de sistemas de información geográfica ofrece una visión rápida y general de los patrones de ocurrencia de atropellos, permitiendo realizar comparaciones y facilitaría la implementación de respuestas a nivel local.

Palabras clave: Sistemas de información geográfica; Análisis espacial; Vehículos a motor; Accidentes de tránsito; Perú (fuente: DeCS BIREME).

\section{GEOGRAPHIC INFORMATION SYSTEMS: PRACTICAL APPLICATION TO STUDY CAR ACCIDENTS INVOLVING PEDESTRIANS IN CERCADO DE LIMA, PERU}

\begin{abstract}
The aim of this study was to geospatially explore the occurrence rates of car accidents involving pedestrians in Cercado de Lima (Lima District), Peru. Car accidents involving pedestrians recorded in the 2015 National Police Station Census of the National Statistics and Information Institute were described and georeferenced. Subsequently, a Kernel Density analysis was carried out to locate areas with high, medium, and low density events. Records of 171 car accidents involving pedestrians were studied: the types of vehicles involved were automobiles (56.7\%) and smaller vehicles $(22.8 \%)$. The highest percentage of car accidents involving pedestrians $(38.6 \%$ ) took place between 12:00 p.m. and 5:00 p.m. There were two densely populated areas and two areas with intermediate density for car accidents involving pedestrians, locations that were previously reported as critical due to their deficiencies and high probability of traffic accidents. The use of geographic information systems offers a quick overview of the occurrence rates of car accidents involving pedestrians to make comparisons and enable the local implementation of strategies.
\end{abstract}

Key words: Geographic information systems; Spatial analysis; Motor vehicles; Accidents, traffic; Peru (source: MeSH NLM).

\section{INTRODUCCIÓN}

Las muertes por lesiones no intencionales constituyen un serio problema de salud pública a nivel mundial. En el año 2010 , este tipo de muertes representaron el 9,6\%
(5,1 millones) del total de fallecimientos en el mundo, de los cuales 1,3 millones fueron debidos a accidentes de tránsito (AT), y el $62,3 \%$ de estas últimas debido a atropellos (1). A nivel mundial, se calcula que el costo generado por AT asciende a 518 mil millones de dólares,

\footnotetext{
Universidad de Buenos Aires. Buenos Aires, Argentina.

Instituto de Investigación, Universidad Católica los Ángeles de Chimbote. Ancash, Perú.

Universidad Nacional San Luis Gonzaga. Ica, Perú.

Universidad San Ignacio de Loyola. Lima, Perú.

Médico cirujano; ${ }^{\mathrm{b}}$ magíster en Gestión y Políticas Públicas; ${ }^{\mathrm{c}}$ cirujano dentista; ${ }^{\mathrm{d}}$ estudiante de Medicina; ${ }^{\mathrm{e}}$ médico veterinario, ${ }^{\mathrm{f}}$ magíster en Gestión de Información y del Conocimiento; ${ }^{\mathrm{g}}$ médico; ${ }^{\mathrm{h}}$ especialista en Epidemiología.

Recibido: 11/02/2016 Aprobado: 07/09/2016
} 
de los cuales 65 mil millones corresponderían a países de bajos y medianos ingresos ${ }^{(2)}$, que es donde ocurren más del $90 \%$ de las muertes relacionadas con AT ${ }^{(3)}$.

En el Perú, tanto la incidencia de AT como su mortalidad y consecuencias atribuidas han aumentado a través de los años. Solo en la región Lima la incidencia aumentó a más del doble entre 1993 a 2008, donde los niños son el grupo con mayor incidencia y fatalidad por AT (4); asimismo, para el periodo 1994-2013, el 61\% de AT en el país ocurrieron en esta región (la más poblada del Perú) ${ }^{(5)}$. Esta tendencia ascendente hizo que para el año 2012, a nivel nacional, los AT representaran la primera causa de años de vida saludable perdidos en varones, con una razón de 10,3 años por cada mil habitantes ${ }^{(6)}$ y para el 2013 ocupen la tercera posición entre las principales causas de años de vida perdidos por muerte prematura ${ }^{(7)}$. Además, se estima que los costos anuales por daños físicos ascenderían a mil millones de dólares, siendo el costo anual, solo en rehabilitación, de aproximadamente $0,12 \%$ del producto bruto interno ${ }^{(8)}$. La existencia de estas cifras merece especial atención y deben ser tomadas en cuenta para redirigir esfuerzos hacia la implementación de estrategias enfocadas a la prevención de AT a nivel nacional.

La disponibilidad de información adecuada y oportuna acerca de la ocurrencia, distribución y factores de riesgo para los AT ayudaría a definir y priorizar un conjunto de estrategias preventivas en áreas con alta incidencia. Los sistemas de información geográfica (SIG) han demostrado utilidad al momento de informar los patrones de ocurrencia de eventos en distintos espacios de un área geográfica ${ }^{(9)}$. Ya se vienen aplicando en la prevención de lesiones, como es el caso de AT, con el propósito de obtener, complementar y/o profundizar el análisis y reporte de estos eventos (10-11). No obstante, estas tecnologías aún son poco empleadas en el Perú, por lo que su utilización contribuiría a prevenir y abordar los AT para disminuir su impacto en la sociedad, especialmente en zonas como Lima, caracterizadas por altos flujos y condiciones adversas de tránsito.

En ese sentido, decidimos realizar un análisis del Censo Nacional de Comisarías 2015, incluyendo los datos de atropellos en el distrito de Lima (también conocido como Cercado de Lima, zona con alto tránsito vehicular y peatonal) con el objetivo de explorar las características y la distribución geoespacial de posibles patrones de ocurrencia de atropellos que puedan ser utilizados en la prevención de desenlaces negativos ocasionados por los AT.

\section{EL ESTUDIO}

Se realizó un análisis descriptivo y espacial de atropellos ocurridos en el Cercado de Lima, ubicado en el centro de la ciudad, con coordenadas $12^{\circ} 02^{\prime} 48^{\prime \prime}$ latitud sur y $77^{\circ} 01^{\prime} 56$ ' longitud oeste, $161 \mathrm{~m}$ de altitud sobre el nivel del mar, superficie de $21,98 \mathrm{~km}^{2}$ y una densidad poblacional de 12366 habitantes $/ \mathrm{km}^{2}$.

Se usó como fuente de información el Censo Nacional de Comisarías 2015, realizado por el Instituto Nacional de Estadística e Informática (INEI) del Perú, en coordinación con el Ministerio de Economía y Finanzas, y el Ministerio del Interior. El censo tuvo como objetivo disponer de una base de datos con información estadística confiable y oportuna sobre el estado actual de los locales donde funcionan las comisarías, relacionado con su infraestructura, equipamiento, seguridad ciudadana; así como también para obtener información del total de denuncias y características de los AT registrados en libros de ocurrencias y sistemas informatizados de denuncias policiales de las comisarías durante el 2014.

El análisis descriptivo utilizó el módulo de AT del censo (http://iinei.inei.gob.pe/microdatos/). En un inicio, se procedió a importar la base de datos de la encuesta en el programa Microsoft Excel $尺 2013$ (Microsoft, Redmond, WA) para luego filtrar los registros y seleccionar los atropellos ocurridos en el 2014 dentro de las jurisdicciones de las diez comisarías existentes en el Cercado de Lima. Los resultados se consolidaron en una hoja de cálculo para la elaboración de medidas de resumen (frecuencias absolutas y proporciones) de las características principales de los atropellos ocurridos (tipo de accidente, horario, tipo de transporte, tipo de vía, consecuencias del accidente, y tipo de vehículo).

Seguidamente, se realizó la georreferenciación utilizando para ello la variable "lugar del accidente". Mediante Google Maps se localizó cada una de las direcciones especificadas en la hoja de cálculo, y se lograron identificar 160, representadas por puntos negros (Figura 1). En los otros once casos la información registraba el nombre de la calle o avenida sin otras especificaciones, por lo que fueron excluidos de esta representación. Para cada punto localizado, se extrajeron las coordenadas de latitud y longitud que se agregaron a la hoja de cálculo.

El análisis espacial se realizó con el programa ArcGIS Desktop versión 10.4 (ESRI Inc., Redlands, CA, USA). Se proyectó cada punto en formato shapefile (.shp) y utilizando la técnica denominada análisis Kernel Density (densidad Kernel gaussiana por $\mathrm{km}^{2}$ ) con ancho de banda predeterminado y tamaño de celda de 0,00018; se estimó la relación de los puntos para clasificar en intervalos iguales aquellas áreas con alta (áreas rojas), mediana (áreas amarillas) y baja densidad (áreas azules) de eventos. Esta técnica analiza la relación entre varios puntos o eventos geográficos (por ejemplo, puntos georreferenciados de atropellos), de manera que los puntos más cercanos generan áreas de mayor 


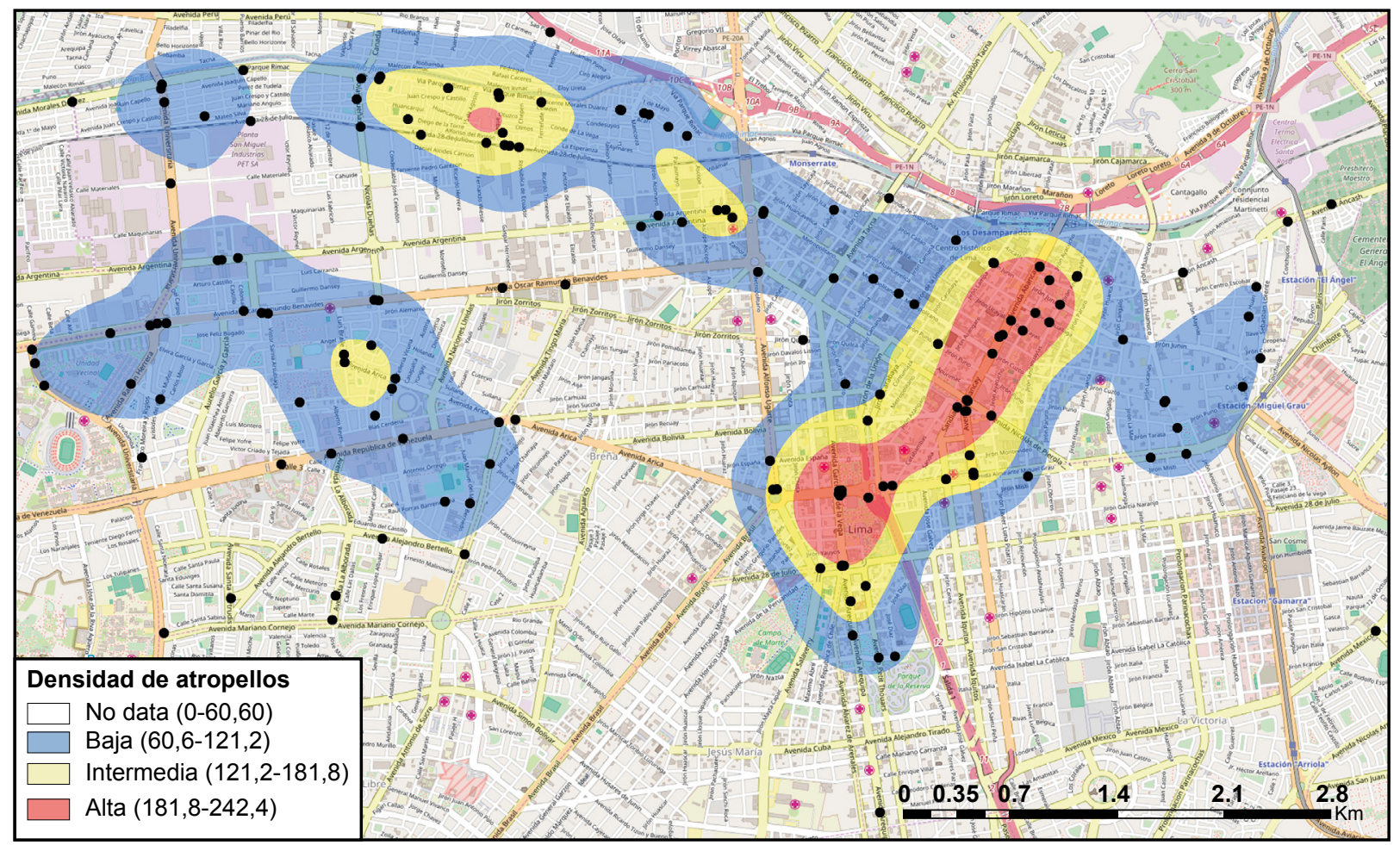

Figura 1. Análisis de densidad Kernel $\left(\mathrm{km}^{2}\right)$ de los puntos georreferenciados de atropellos ocurridos en el Cercado de Lima utilizando OpenStreetMap como mapa de base - Censo Nacional de Comisarías 2015.

frecuencia (densidad) dentro de un área determinada. Se generó una capa de densidad con los 160 puntos. Asimismo, para una mayor visualización de los eventos según condiciones horarias, se generaron dos capas de densidad, una con los accidentes ocurridos entre las 06.00 y $17.00 \mathrm{~h}$ (horario diurno) con 100 accidentes, y otra con 60 puntos de accidentes ocurridos entre las 18.00 y $05.00 \mathrm{~h}$ (horario nocturno). Los shapefiles creados fueron vinculados y proyectados a mapas de base de OpenStreetMap.

Este estudio no requirió la aprobación de un comité de ética por tratarse de un análisis secundario de una encuesta cuyos datos son de libre acceso, dominio público y no permiten identificar a los involucrados en los AT.

\section{HALLAZGOS}

Se encontraron 171 registros de atropellos, en el 13,5\% ocurrió fuga del vehículo. Según horarios de ocurrencia, el mayor porcentaje de atropellos $(38,6 \%)$ sucedió entre las 12.00 y $17.00 \mathrm{~h}$. Los tipos de transporte involucrados con mayor frecuencia fueron los vehículos particulares $(56,7 \%)$ y públicos $(15,2 \%)$. Los tipos de vía con mayor ocurrencia fueron las avenidas $(70,8 \%)$, seguido de calles o jirones $(28,7 \%)$. El mayor número de atropellos presentó consecuencias no fatales $(98,2 \%)$. Con respecto al tipo de vehículo involucrado, en el 56,7\% de casos participaron automóviles (Tabla 1).
Tabla 1. Características principales de los atropellos ocurridos en el Cercado de Lima, Censo Nacional de Comisarías 2015

\begin{tabular}{|c|c|c|}
\hline \multirow{2}{*}{ Características } & \multicolumn{2}{|c|}{ Accidentes } \\
\hline & $\mathbf{N}$ & $(\%)$ \\
\hline \multicolumn{3}{|l|}{ Tipo de accidente } \\
\hline Atropello & 148 & $(86,5)$ \\
\hline Atropello y fuga & 23 & $(13,5)$ \\
\hline \multicolumn{3}{|l|}{ Horario (horas) } \\
\hline 0.00 a 05.59 & 10 & $(5,8)$ \\
\hline 06.00 a 11.59 & 39 & $(22,8)$ \\
\hline 12.00 a 17.59 & 66 & $(38,6)$ \\
\hline 18.00 a 23.59 & 56 & $(32,7)$ \\
\hline \multicolumn{3}{|l|}{ Tipo de transporte } \\
\hline Público & 26 & $(15,2)$ \\
\hline Particular & 97 & $(56,7)$ \\
\hline No identificado & 48 & $(28,1)$ \\
\hline \multicolumn{3}{|l|}{ Tipo de vía } \\
\hline Autopista* & 1 & $(0,6)$ \\
\hline Avenida & 121 & $(70,8)$ \\
\hline Calle o Jirón & 49 & $(28,7)$ \\
\hline \multicolumn{3}{|l|}{ Consecuencia del accidente } \\
\hline Fatal & 2 & $(1,2)$ \\
\hline No fatal & 168 & $(98,2)$ \\
\hline Solo daños materiales & 1 & $(0,6)$ \\
\hline \multicolumn{3}{|l|}{ Tipo de vehículo } \\
\hline Menor** & 39 & $(22,8)$ \\
\hline Automóvil & 97 & $(56,7)$ \\
\hline Otros & 35 & $(20,5)$ \\
\hline
\end{tabular}


Con respecto al análisis espacial, la Figura 1 muestra en la densidad Kernel dos zonas con alta densidad de ocurrencia de atropellos (áreas rojas), una de ellas se ubica en los alrededores de las avenidas Morales Duarez y Enrique Meiggs, y la otra, de mayor amplitud, alrededor de la avenida Abancay y su intersección con la avenida Nicolás de Piérola; así como sobre la intersección de la avenida Inca Garcilaso de la Vega, la avenida 9 de diciembre y el óvalo Miguel Grau. De igual manera, el análisis determinó dos zonas de densidad intermedia (áreas amarillas), una de ellas ubicada sobre la avenida Argentina en su porción cercana a la plaza Ramón Castilla y la otra en la intersección entre las avenidas Arica y Luis Braille.

En las Figuras 2 y 3 , se grafican las densidades de atropellos según el horario de ocurrencia. Durante el día se observaron dos áreas de alta densidad, una de ellas la avenida Abancay en sus intersecciones con los jirones Junín y Huallaga, y la otra en la intersección entre las avenidas Inca Garcilaso de la Vega y 9 de diciembre; ambas áreas rojas se encuentran rodeadas de áreas amarillas de densidad intermedia. Además, se registraron tres áreas con densidad intermedia de atropellos sobre las avenidas Morales Duarez y Enrique Meiggs, en su cercanía con sus intersecciones con la avenida Nicolás Dueñas; sobre la intersección de la plaza Ramón Castilla y la avenida Argentina y el área entre las avenidas Argentina y Oscar Benavides, cercana a su intersección con la avenida Universitaria. Durante la noche solo se observa un área de alta densidad de atropellos, la cual está ubicada alrededor de la intersección entre las avenidas Abancay y Nicolás de Piérola. Adicionalmente, se aprecia un área de densidad intermedia entre las avenidas Morales Duarez y Enrique Meiggs.

\section{DISCUSIÓN}

Mediante el análisis espacial aplicado en nuestro estudio se lograron identificar áreas con mayor concentración de atropellos en el Cercado de Lima durante el 2014, así como las avenidas, calles o jirones comprendidos dentro de estas.

El Cercado de Lima, al ser una zona urbano-comercial, se caracteriza por su tránsito caótico producto de la gran cantidad de vehículos que circulan por su área, así como de un considerable número de peatones que se movilizan por esta zona debido a sus atractivos turísticos, presencia de centros laborales y locales comerciales; todos estos representan factores de riesgo para la ocurrencia de atropellos ${ }^{(5)}$. Un estudio realizado por el Ministerio de Transportes y Comunicaciones del Perú

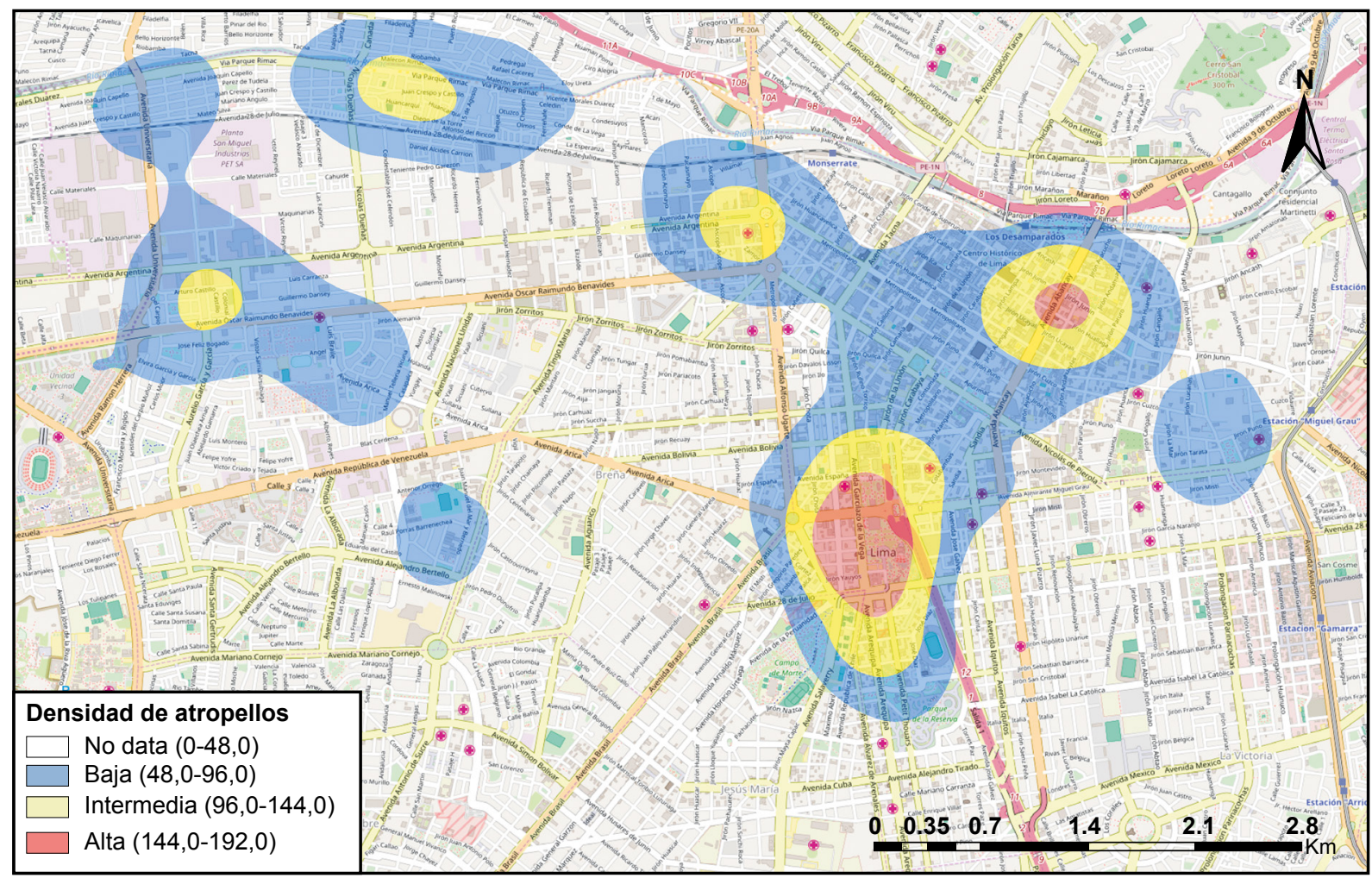

Figura 2. Análisis de densidad Kernel $\left(\mathrm{km}^{2}\right)$ de los puntos georreferenciados de atropellos ocurridos durante el día en el Cercado de Lima utilizando OpenStreetMap como mapa de base - Censo Nacional de Comisarías 2015. 


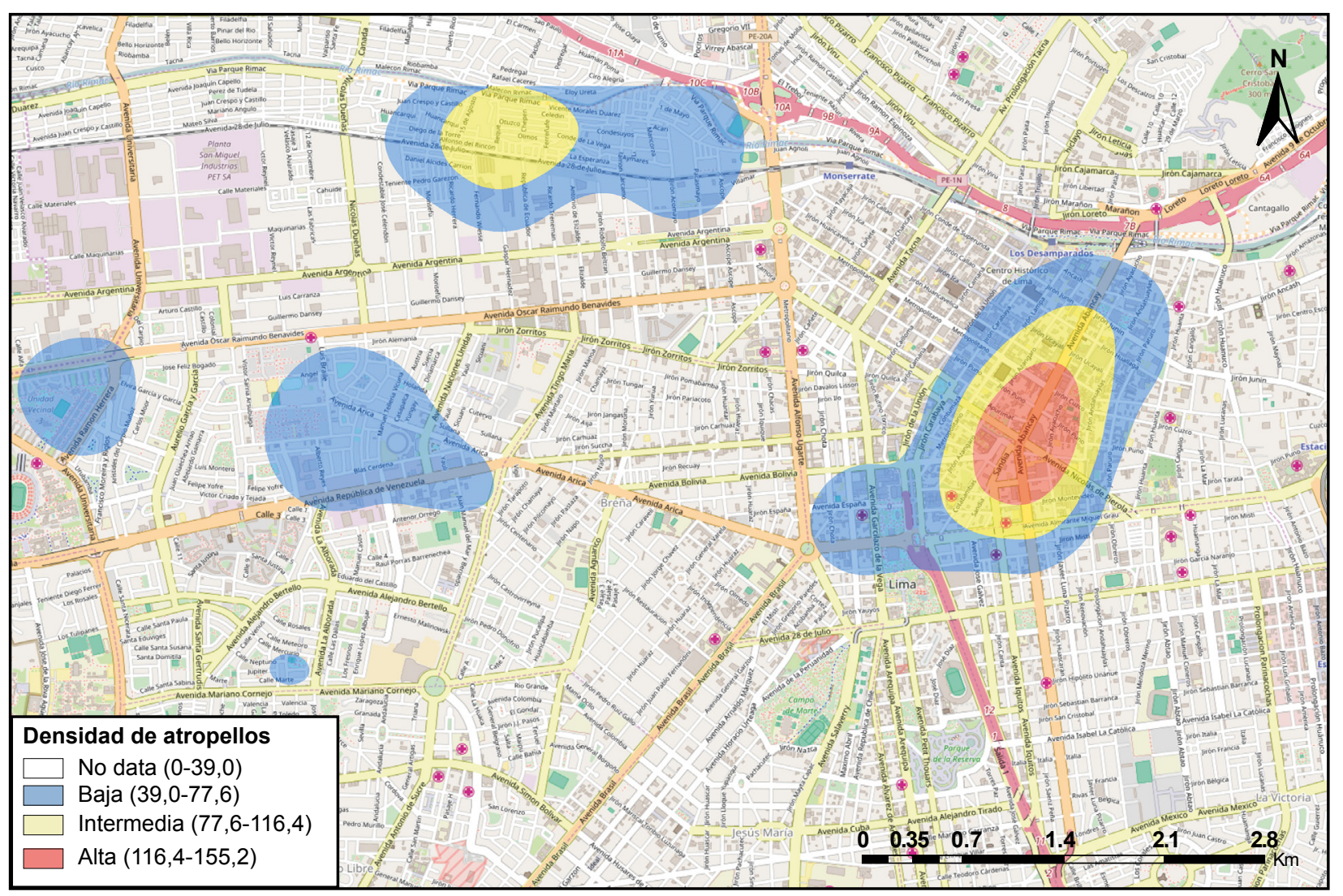

Figura 3. Análisis de densidad Kernel $\left(\mathrm{km}^{2}\right)$ de los puntos georreferenciados de atropellos ocurridos durante la noche en el Cercado de Lima utilizando OpenStreetMap como mapa de base - Censo Nacional de Comisarías 2015.

detectó 205 puntos negros en esta área entre los años 2012-2015 (12); sin embargo, hace falta complementar esta información con el empleo de análisis espaciales, los cuales han resultado ser útiles en diversos países para la detección de sitios de intervención ${ }^{(13-14)}$.

La alta ocurrencia de atropellos en la intersección de la avenida Inca Garcilaso de la Vega y el Paseo Colón, además de los hallados en la avenida Abancay y los reportados en las avenidas Morales Duarez y Enrique Meiggs, son áreas que coinciden con las identificadas en los informes técnicos realizados entre 2011 y 2015 por la Defensoría del Pueblo sobre puntos críticos de AT en Lima y Callao, en donde reportan que existe la necesidad de dar mantenimiento a la infraestructura vial; demarcar los cruces peatonales; necesidad de nuevas señalizaciones, mejorar las estaciones de embarque, desembarque y accesibilidad a rampas para personas con discapacidad ${ }^{(5,15)}$. Consideramos que estas recomendaciones junto a otras medidas que han demostrado ser efectivas como el uso de material audiovisual con contenido preventivo-promocional, distribución permanente y estratégica de policías de tránsito en los horarios de mayor ocurrencia de atropellos, empleo de elementos de reducción de la velocidad y del tránsito peatonal, implementación de restricciones horarias de expendio de bebidas alcohólicas y dotación efectiva de policías de tránsito en las zonas de riesgo, podrían contribuir a la disminución de los AT y atropellos en estas localizaciones ${ }^{(16,17)}$.

Nuestro trabajo presenta limitaciones al emplear datos secundarios, debido a la calidad y precisión de los mismos. En relación al Censo Nacional de Comisarías 2015, al recabarse la información de fuentes policiales no podemos asegurar un nivel de representatividad total de los AT, ya que en algunos casos pueden no haberse reportado por la poca severidad de las lesiones o desinterés de los afectados, o por un registro inadecuado de la información. Por ejemplo, el Consejo Nacional de Seguridad Vial señala que existe divergencia en la información brindada en fuentes policiales sobre AT en el Perú, encontrando que existe hasta un $9 \%$ de reportes que no consiguen la clase de evento (18); estas diferencias pueden asociarse a los distintos organismos involucrados en esta tarea ${ }^{(19)}$.

Por otro lado, la técnica utilizada (densidad Kernel) es una prueba no paramétrica basada en la mayor frecuencia de ocurrencia de eventos, sin establecer pruebas de significancia estadística que permitan establecer con 
certeza si los patrones encontrados son aleatorios; sin embargo, esta es una técnica ampliamente utilizada en los estudios basados en SIG. A pesar de estas limitaciones, consideramos que nuestros hallazgos complementan los pocos estudios realizados que han descrito in situ los puntos críticos de AT en el Cercado de Lima, brindando una perspectiva geoespacial de los mismos.

Los resultados de este estudio demuestran la utilidad de los SIG son útiles para lograr una visión rápida y general de los patrones de ocurrencia de atropellos en el Cercado de Lima, lo cual servirá para implementar respuestas inmediatas y/o medidas preventivas. Este tipo de herramientas de seguimiento de la información tendrían mayor utilidad en áreas de la selva peruana en donde el uso masivo de motos lineales hace que las lesiones por AT tengan peores consecuencias ${ }^{(20)}$.
De igual manera, los resultados obtenidos servirán para comparar los patrones actuales de atropellos con escenarios futuros. Finalmente, es prioritario que se implementen estrategias orientadas a recopilar datos precisos sobre los AT en el Perú que generen información sistematizada y oportuna por parte de las instituciones involucradas en el estudio, análisis e investigación de los AT que sirva para la toma efectiva de decisiones a nivel local, regional y nacional.

Fuentes de financiamiento: autofinanciado.

Conflictos de interés: los autores declaran no tener conflictos de interés.

Contribución de autoría: AHV, DA y RMCh participaron en la concepción, diseño del estudio y recolección de los datos. AHV realizó el control de calidad y el análisis de los datos. Todos los autores participaron en la interpretación de los datos, redacción del artículo, revisión y aprobación de la versión final.

\section{REFERENCIAS BIBLIOGRÁFICAS}

1. Lozano R, Naghavi M, Foreman K, Lim S, Shibuya K, Aboyans V, et al. Global and regional mortality from 235 causes of death for 20 age groups in 1990 and 2010: a systematic analysis for the Global Burden of Disease Study 2010. Lancet. 2012;380(9859):2095-128. doi: 10.1016/S0140-6736(12)61728-0

2. Organización Mundial de la Salud. Informe mundial sobre prevención de los traumatismos causados por el tránsito [Internet]. Geneva, Switzerland: OMS; 2004 [citado el 25 de febrero de 2016]. Disponible en: http://apps.who.int/iris/bitstream/10665/42926/1/9243591312. pdf

3. Organización Mundial de la Salud. Lesiones causadas por el tránsito [Internet]. Ginebra, Suiza: OMS; 2015 [ciitado el 17 de marzo de 2016]. Disponible en: http://www.who.int/mediacentre/factsheets/fs358/es/

4. Miranda JJ, Lopez-Rivera LA, Quistberg DA, Rosales-Mayor E, Gianella C, Paca-Palao A, et al. Epidemiology of road traffic incidents in Peru 1973-2008: incidence, mortality, and fatality. PloS One. 2014;9(6):e99662. doi: 10.1371/ journal.pone.0099662

5. Defensoría del Pueblo. Supervisión de las condiciones de infraestructura vial en puntos críticos de accidentes de tránsito en los distritos de Lima y Callao [Internet]. Lima, Perú 2015 [citado el 20 de diciembre de 2015]. Disponible en: http://www.defensoria. gob.pe/modules/Downloads/ informes/varios/2015/IA-003-2015DP-AMASPPI-SP.pdf

6. Ministerio de Salud. Carga de enfermedad en el Perú, estimación de los años de vida saludables perdidos 2012 [Internet]. Lima, Perú: MINSA; 2014 [citado el 04 de marzo de 2016]. Disponible en: http://www. dge.gob.pe/portal/docs/tools/ Cargaenfermedad2012.pdf.

7. Institute for Health Metrics and Evaluation. [Internet]. Seattle, USA: University of Washington; 2016 [citado el 25 de febrero de 2016]. Disponible en: http://www. healthdata.org/peru.

8. Ministerio de Salud. Plan nacional de la estrategia sanitaria nacional de accidentes de tránsitoESNAT-2009-2012. [Internet]. Lima, Perú: MINSA; 2009 [citado el 29 de febrero de 2016]. Disponible en: http://bvs.minsa.gob.pe/local/ MINSA/718_MINSA1059.pdf.

9. Lin YP, Chu HJ, Wu CF, Chang TK, Chen CY. Hotspot analysis of spatial environmental pollutants using kernel density estimation and geostatistical techniques. Int J Environ Res Public Health. 2011;8(1):75-88. doi: 10.3390/ijerph8010075

10. Bell N, Schuurman N. GIS and injury prevention and control: history, challenges, and opportunities. Int J Environ Res Public Health. 2010;7(3):1002-17. doi: 10.3390/ ijerph7031002

11. De Andrade L, Vissoci JR, Rodrigues CG, Finato K, Carvalho E, Pietrobon $\mathrm{R}$, et al. Brazilian Road Traffic Fatalities: A Spatial and Environmental Analysis. PloS One. 2014;9(1):e87244. doi: 10.1371/journal.pone.0087244

12. Ministerio de Transportes y Comunicaciones. Detección, priorización y caracterización de puntos negros en 5 ciudades principales del Perú. Proyecto de Identificación y Priorización de Puntos Negros [Internet]. Lima, Perú 2015 [citado el 20 de diciembre de 2015]. Disponible en: http://www.mtc.gob. pe/transportes/terrestre/documentos/ estudio_puntos_negros.pdf.

13. Blazquez CA, Celis MS. A spatial and temporal analysis of child pedestrian crashes in Santiago, Chile. Accid Anal Prev. 2013;50:304-11. doi: 10.1016/j. aap.2012.05.001

14. Gómez-Barroso D, López-Cuadrado T, Llácer A, Palmera Suárez R, Fernández-Cuenca R. Análisis espacial 
de los accidentes de tráfico con víctimas mortales en carretera en España, 20082011. Gac Sanit 2015;29 Supl 1:24-9. doi: 10.1016/j.gaceta.2015.02.009

15. Defensoría del Pueblo. Informe $\mathrm{N}^{\circ}$ 008-2012-DP/AMASPPI.SP Seguridad vial y puntos negros en Lima Metropolitana [Internet]. Lima, Perú 2012 [citado el 01 de agosto de 2016]. Disponible en: http://www2.congreso.gob.pe/sicr/cendocbib/con4_uibd. nsf/92E37EC463E8C92E05257EBB006EB2F0/\$FILE/Informe-0082012-DP-AMASPPI-SP.pdf.

16. Málaga $H$. Medidas y estrategias para la prevención y control de los accidentes de tránsito: experiencia peruana por niveles de prevención. Rev Peru Med Exp
Salud Publica. 2010;27(2):231-6. doi: 10.1590/S1726-46342010000200011

17. Rodríguez-Hernández JM, Campuzano-Rincón JC. Medidas de prevención primaria para controlar lesiones y muertes en peatones y fomentar la seguridad vial. Revista Salud Publica (Bogota). 2010;12(3):497-509.

18. Consejo Nacional de Seguridad Vial. Plan Nacional de Seguridad Vial 20152024 [Internet]. Lima, Perú 2015 [citado el 05 de agosto de 2016]. Disponible en: https://www.mtc.gob.pe/cnsv/ Proyecto\%20del\%20Plan\%20Nacional\%20de\%20Seguridad\%20Vial\%20 2015_2024.pdf.

19. Miranda JJ, Paca-Palao A, Najarro L, Rosales-Mayor E, Luna D, Lopez L, et al. Evaluación situacional, estructura, dinámica y monitoreo de los sistemas de información en accidentes de tránsito en el Perú - 2009. Rev Peru Med Exp Salud Publica. 2010;27(2):273-87. doi: 10.1590/ S0124-00642010000300015

20. Berecki-Gisolf J, Yiengprugsawan V, Kelly M, McClure R, Seubsman S, Sleigh A, et al. The impact of the thai motorcycle transition on road traffic injury: Thai cohort study results. PloS One. 2015;10(3): e0120617. doi: 10.1371/journal.pone.0120617

Correspondencia: Akram Abdul Hernández Vásquez

Dirección: Calle Gavilán 3856, Ciudad Autónoma de Buenos Aires, Argentina Teléfono: (0054) 91122981572

Correo electrónico: akram.hernandez.v@upch.pe

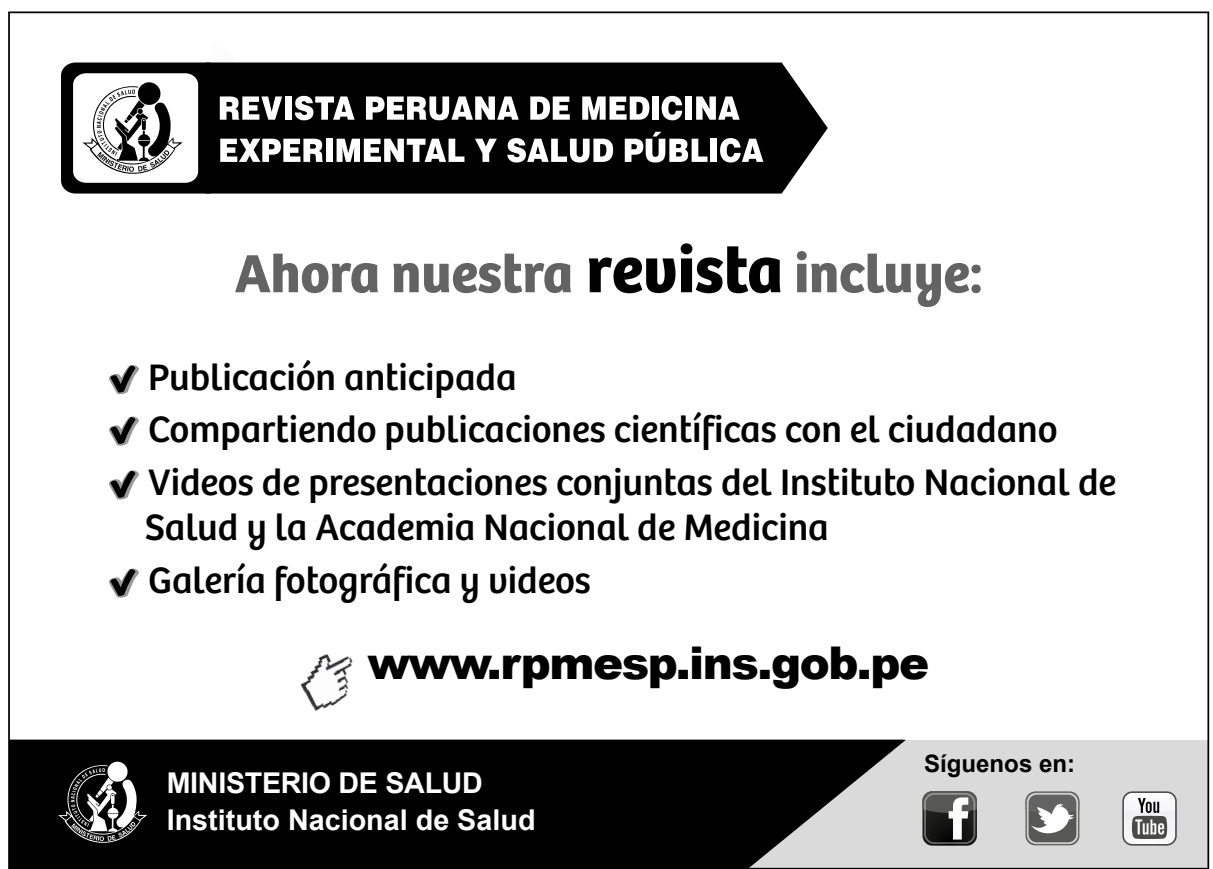

